\title{
16 \\ ENFORCING INTEGRITY WHILE MAINTAINING SECRECY
}

\author{
Donald G. Marks
}

\begin{abstract}
We consider the role of constraints in maintaining both secrecy and integrity in a multilevel secure database. In a multilevel database, certain integrity and classification constraints create a secrecy problem since data additions, deletions or modifications require accessing data at higher levels. In many cases, however, these constraints may be approximated by a series of simpler constraints, called level-valid constraints. Level-valid constraints do not require access to any data that is classified higher than the data to be modified. Moreover, they meet the integrity requirements since any database state that satisfies the level-valid constraints also satisfies the multilevel constraints. Simple tests are developed to ensure the validity of proposed level-valid constraints and these constraints are derived for common cases of multilevel constraints.
\end{abstract}

Keywords: Integrity, secrecy

\section{INTRODUCTION}

Consistency, or data integrity, in databases is usually achieved by associating with each database a set of constraints. The database management system (DBMS) has the responsibility to ensure that these constraints are satisfied by the database state at all times. Generally, this is done by checking the constraint for each tuple that is added, deleted or modified. A multilevel secure (MLS) DBMS has the additional responsibility of preventing improper disclosure ${ }^{1}$ of information either by direct or indirect means. It is well known that there are inherent conflicts in MLS databases between the secrecy requirements and certain types of integrity constraints [3]. In particular, it appears impossible to enforce certain integrity constraints without violating the secrecy requirements.

The original version of this chapter was revised: The copyright line was incorrect. This has been corrected. The Erratum to this chapter is available at DOI: 10.1007/978-0-387-35508-5_22 
Since the enforcement of multilevel constraints involves a trade-off between secrecy and integrity, the usual approach is to accept one or the other.

In this study, we show how it is often possible to maintain secrecy while still enforcing integrity. The approach taken will be to translate the original multilevel constraint, whose satisfaction requires the MLS DBMS to access both High and Low data, into a collection of level-valid ${ }^{2}$ constraints. Each level-valid constraint has a fixed security level associated with it and is evaluated by referencing only data at or below that level. In a sense, then, we polyinstantiate the constraint rather than the tuple. That is, instead of having one constraint that applies to all levels, each level will have its own constraint that only applies to that level.

More formally, suppose we have a database state, $D$ and a multilevel constraint $M$. To enforce both the constraint and the security policy, we wish to replace $M$ by a collection of level-valid constraints $L$, such that:

$$
L=\{L 1, L 2, \ldots L n, M\} \text { (where } L_{i} \text { and } L_{j} \text { are not necessarily unique) }
$$

1. Each $L_{j}$ in $L$ is a level-valid constraint at level $j$, and

2. If $D$ satisfies level-valid constraint $L_{j}$, then $\mathrm{D}$ also satisfies $L_{j+1}$.

At the highest level, the constraint is still $M$, so there may exist database states that are allowable under the original multilevel constraint but not under the derived level-valid ones. Processes allowed to modify the database in a way that meets the original multilevel constraint, but not the appropriate levelvalid constraint, will still have to be trusted at the higher level (note that this higher level constraint is not necessarily $M$ ). Thus, our approach is still a compromise between security and integrity. However, the use of such trusted processes is reduced by this scheme. Intuitively, if the level-valid constraint represents all the pertinent information available at the lower level, the scheme would minimize the use of trusted processes. Actually forming constraints that minimize these trusted processes is still an area of research, however.

\subsection{PREVIOUS WORK}

Within a "multilevel secure" model, as in conventional databases, constraints are a common means of controlling not only integrity, but also access and inference control. Other researchers note the issue of conflict between the multilevel security and database integrity requirements in DBMS design. In particular, [3, 1, 7, 2, and 6], have all addressed this issue. Thuraisingham and Ford [THUR95], Sandhu and Jajodia [9], and Qian [8] have addressed

${ }^{2}$ From [8] 
polyinstantiation (i.e. multiple data instances) issues associated with integrity constraints. Gupta and Widom [4] propose a similar technique for local verification of constraints in distributed databases, although no security issues are addressed. While some of the theoretical and design issues for this approach were discussed in [5], this paper focuses upon the mechanics and the process of translating multi-level constraints into equivalent sets of level-valid constraints.

\subsection{EXAMPLE 1}

Suppose we have the relation: $\operatorname{EMP}($ Name, Salary, Position $)$ c containing the names, salaries, and positions of the employees in a law enforcement agency. There are three positions: patrolman, investigator, and special agent. The constraints are:

1) all patrolman salaries are less than any investigator's salary;

2) all investigator salaries are less than any special agent's salary.

The classification levels are: 1) patrolman records are classified confidential (C); 2) investigator records are classified secret (S);3) special agent records are classified top secret (TS). Subjects at one level are not allowed to read information at a higher level, nor are they allowed to write at any other level.

If a new patrolman is hired, that record is automatically classified $\mathrm{C}$, and inserted by a C-level subject. The database must decide if this is allowed. At the $\mathrm{C}$ level, the level-valid constraint is:

$L_{C}$ : New patrolman salary must be less than or equal to the highest existing patrolman salary.

This constraint is sufficient to guarantee that a new tuple does not invalidate the original constraint.

The highest cleared category, special agent, has access to all lower classified information and needs no special constraint, so:

$L_{T S}$ : New special agent salary must be higher than the highest existing investigator salary.

Finally, if a subject attempts to write a new investigator tuple, there are two checks that must be performed: the salary value must be higher than any patrolman, but lower than any special agent. This will require a conjunctive constraint to specify both conditions as follows: 
$L_{S}$ : New investigator salary must be less than the highest existing investigator salary, and greater than the highest paid patrolman.

This constraint is sufficient to guarantee that a new tuple satisfies both of the original multilevel constraints.

This example illustrates integrity constraints which are affected only by insert operations and are sufficient to enforce the original multi-level constraint. Note that constraints that are enforced during an insert operation may be different from the constraints that are enforced during a delete operation.

The next sections will formalize and generalize the approach to provide methods of forming these level-valid constraints as well as assurances of their correctness.

\section{THE GENERAL SOLUTION}

\section{$2.1 \quad$ NOTATION}

The first necessary step is to define a specific formalism for constraints. Constraints are generally formed by comparing characteristics (given by a function) of two sets of data (given by a database view). This can be formalized in the following definition.

Definition of Constraint: a constraint is an expression of the form: $f_{1}\left(q_{1}(R)\right) \Theta f_{2}\left(q_{2}(R)\right)$. Which is true for any valid database state.

Where: $q_{1}, q_{2}$ are database views on the relation $\mathrm{R} ; f_{1}, f_{2}$ are functions defined on the data revealed by the database views and $\Theta$ defines a partial order relating the values derived by $f_{1}, f_{2}$. To avoid the complexities of considering the database representation, we adopt the universal relation viewpoint, so that the relation $R$ is equivalent to the entire database.

The functions are defined using mathematical notation, i.e. $f(x)=y$, where $x$ is the argument of the function. Note that $f_{1}, f_{2}$ may be any user defined functions, including those implemented by means of triggers, procedures or object oriented methods. Functions however, are limited to acting upon the information included in the view defined as their argument. Similarily, $\Theta$ may be any partial order, including functional dependence from relational database theory. However, commercial database systems only allow for a few comparators so in this study we assume that $f_{1}, f_{2}$ yield either a numerical value or a set of string values. We also assume $\Theta$ is one of the standard comparators; ( $>$; $<; \leq ; \geq ; \Rightarrow$ ) for numeric values, or the subset inclusion operator " $\subseteq$ ", or its 
inverse " $\supseteq$ " for string values (or sets of numerics). In later discussions, $\Omega$ will denote " $\Theta$ or $=$ ", so if $\Theta \equiv<$, then $\Omega \equiv \leq$.

For any multi-level database, some of the data may be viewed by a subject cleared to level $L$, namely that information classified no higher than level $L$. We will denote this restricted view by the subscript $L$. Therefore, $R_{L}$ will denote that subset of $R$ which is visible at level $L$.

The function and view based notation presented here is more intuitive than previously proposed notations and allows the database itself to evaluate the constraints. It maintains separation of important concepts (views, functions, and tests), allows for multiple aggregate functions, and is not restricted to using base relations or conjunctions of simple selects. In our notation, the constraint "all patrolman salaries are less than any investigator's salary" becomes:

\section{MAX(select Salary from EMP where Position = 'patrolman') $\leq$ \\ MIN(select Salary from EMP where position = 'investigator').}

More complicated constraints may require the following extension:

Definition 2: A complex constraint is an expression of the form $I C_{i}$ AND $I C_{j}$

where either both $I C_{i}$ and $I C_{j}$ are simple constraints as defined in Definition 1 , or $I C_{i}$ is a simple constraint and $I C_{j}$ is a complex constraint.

\subsection{SUFFICIENCY}

The critical feature of this notation is the fact that it provides us with a way to order the relations using the comparator $\Theta$. The fact that the relations are ordered may allow us to derive some simple tests for determining if a tuple may be added to (or deleted from, or modified in) the database. Theorem 1 uses this ordering to establish our a testable condition.

Theorem 1 - Tuple Addition: Given a constraint, $f_{1}\left(q_{1}(R)\right) \Theta f_{2}\left(q_{2}(R)\right)$, which is known to be satisfied by the current state of the database, and a tuple, $t$, satisfying the following two conditions:

(1) $f_{1}\left(q_{1}(R \bigcup t)\right) \Omega f_{1}\left(q_{1}(R)\right)$ and

(2) $f_{2}\left(q_{2}(R)\right) \Omega f_{2}\left(q_{2}(R \bigcup t)\right)$,

then the database will still satisfy the constraint after $t$ is added.

[Theorem 1 may be modified to address delete and modify in a straight- 
forward manner.]

Theorem 1 specifies two conditions, one for each expression in the constraint. We can therefore define two sets of valid tuples, one for each condition. Those tuples in both sets may be added to the relation and still satisfy the original constraint.

[For real-life complex databases, Theorem 1 may have to be implemented using "before and after images". The values of $f_{1}\left(q_{1}(R \bigcup t)\right)$ and $f_{2}\left(q_{2}(R \bigcup t)\right)$ may need to be calculated from the "after" image, that is, after $\mathrm{t}$ is inserted and committed, to the database. Otherwise there may be additional assertions, triggers, constraints and procedures that would change other data in the database view.]

Fortunately, in many cases of practical interest, a substantial number of tuples are in both sets. It is even common for a tuple to influence only one of the conditions in Theorem 1, while the other condition is satisfied by all tuples. For example, if $f_{2}(R)$ is equal to a constant, $k$, then it is true that $f_{2}\left(q_{2}(R)\right)=f_{2}\left(q_{2}(R \bigcup t)\right)=k$, regardless of what tuple $t$ is added to the database. In such cases, tuples only influence one condition, so the conjunction does not present a serious problem.

Example 2. Consider the constraint: "all patrolman salaries are less than any investigator's salary". From Theorem 1, it follows that we need to satisfy the two conditions:

1) MAX $\{$ select Salary from $(E M P \bigcup t)$ where Position = patrolman $\}$ $\leq$

MAX $\{$ select Salary from EMP where Position = patrolman $\}$ and 2) MIN $\{$ select Salary from EMP where Position = investigator $\} \leq$ MIN $\{$ select Salary from $(E M P \bigcup t)$ where Position = investigator $\}$.

Patrolman tuples with salaries less than or equal to the present maximum will not change the value of the first condition in (1). All these tuples will therefore satisfy (1). All patrolman tuples will also satisfy (2) since it is unchanged by their addition, regardless of their salary. The addition of an patrolman tuple therefore only requires evaluation of one, level-valid constraint:

$I_{C}:$ "if t.position $=$ patrolman, then t.Salary $\leq \operatorname{MAX}\{$ select Salary from EMP where Position = patrolman $\}$.

Note that, if each tuple affects only one of the views $\left(q_{1}(R)\right)_{L}$ or $\left(q_{2}(R)\right)_{L}$, then: 


\section{$\left(q_{1}(R)\right)_{L} \bigcap\left(q_{2}(R)\right)_{L}=\emptyset$ (Separation Condition)}

and Theorem 1 may be applied at level $L$. This is a very powerful and useful test. It provides a very simple method to determine when the "multilevel" constraint may be separated into distinct classification levels or compartments. Obviously, if the Separation Condition holds, each new tuple will affect, at most, one of the test conditions in Theorem 1. This affected condition is visible at level $L$, and is the only condition that needs to be evaluated in order to prove the continued validity of the multilevel constraint.

\section{COMMON DBMS OPERATIONS}

\subsection{AUTOMATED CONSTRAINT DERIVATION}

The next step is to automatically propose level-valid constraints which may represent sufficient conditions for the addition of (deletion from, modification to) a tuple in a relation. We do not claim to have evaluated every variation of such constraints, nor to find an optimal solution to the problem. We have, however, attempted to partition the problem and devise appropriate rules for each node in the taxonomy. Using the results will require assigning the constraint to the appropriate node and applying the corresponding rules.

To make it possible to automate the derivation of level-valid constraints, we will assume that the addition of a tuple does not affect $f_{2}\left(q_{2}(R)\right)$. We will also assume that the addition or deletion of a tuple does not cause and "cascade" effects due to other triggers or procedures. The technique could be extended to allow for such circumstances, but the results would have to be framed in terms of the less intuitive before and after images. In the following procedures, we are required to compare views at differing classification levels. Since we must do this comparison at the lowest of these levels, some changes must be made to the view definition. In transmogrifying a high level view into a lower level view we may either create a bigger or a smaller view (disjoint views would have no value). Much of the following discussion formalizes this rather simple observation.

The problem is approached on a case-by-case basis. This approach is appropriate since there are only a limited number of database operations and comparators to be analyzed. In commercial databases, choices of functions are limited to the common aggregate functions of COUNT, MAX, MIN, and SUM. The choices for comparators are similarly limited to $<,>, \leq, \geq,=, \supseteq$, or $\subseteq$. Each combination of a database operation and comparator comprises a case. 


\subsection{RELATIONSHIP OF COMPONENTS}

Assume a multilevel constraint $M=f_{1}\left(q_{1}(R)\right) \Theta f_{2}\left(q_{2}(R)\right)$, where the addition of a tuple at level $L, t$, changes the value of $f_{1}\left(q_{1}(R)\right)$ but does not affect $f_{2}\left(q_{2}(R)\right)$. We will derive a view $q_{3}\left(R_{L}\right)$ such that:

$f_{1}\left(q_{1}(R)\right) \Omega f_{1}\left(q_{3}\left(R_{L}\right)\right)$ always holds, and define the level-valid constraint as:

$$
L=f_{1}\left(q_{3}\left(R_{L}\right)\right) \Omega f_{2}\left(q_{2}(R)\right)_{L}
$$

Then, by transitivity, if $L$ holds, $M$ holds. The only unknown is the view definition $q_{3}\left(R_{L}\right)$. Note that the query $q_{3}$ is submitted by a subject at level $L$ and hence can only operate on $R_{L}$ - the restriction of $R$ to level $L$. The query $q_{2}$, however, is submitted by a High cleared subject and so $q_{2}(R)$ may contain attributes classified higher than $L$. In order to compare the two relations, $q_{2}(R)$ must be restricted to level $L$, (i.e. $\left.\left(q_{2}(R)\right)_{L}\right)$. Even though $q_{1}(R)$ and $q_{3}\left(R_{L}\right)$ may have differing attributes, they could contain some common tuples. A tuple in $q_{1}(R)$ will match a tuple in $q_{3}\left(R_{L}\right)$ if the two tuples have identical primary keys and $\left(q_{1}(t)\right) L=q_{3}\left(t_{L}\right)$. We are especially interested in the situation where all tuples in one set are also in the other. Either $q_{3}\left(R_{L}\right)$ or $q_{1}(R)$ could be the larger set (called the superset) and have either additional tuples, or longer tuples. There are therefore two generic types of views of interest to us: the superset case where $q_{1}(R) \subseteq\left(q_{3}\left(R_{L}\right)\right.$ and the subset case where $q_{3}\left(R_{L}\right) \subseteq\left(q_{1}(R)\right.$.

Superset Views: $q_{1}(R) \subseteq\left(q_{3}\left(R_{L}\right)\right)$. All tuples in the multilevel view $q_{1}(R)$ are also in $q_{3}\left(R_{L}\right)$, and the level-valid view $q_{3}\left(R_{L}\right)$ may be larger. These types of level-valid constraints are frequently formed by deleting those clauses restricting the view to high classified attributes. Thus the low-classified view "ID number of all SR71 airplanes" is certainly a larger set than the high-classified view "ID number of all SR71 airplanes on spy missions".

Subset Views: $q_{3}\left(R_{L}\right) \subseteq q_{1}(R)$. All tuples in the level-valid view $q_{3}\left(R_{L}\right)$ are also in the multilevel view $\left(q_{1}(R)\right)$, and the multilevel view $q_{1}(R)$ may be larger.

Subset views are related to the aggregation problem where a subset of High information is classified Low either by design or because it is common knowledge. This might occur if the view "ID number, Mission, of all SR71 airplanes" is classified High, but the view "ID number of all SR71 airplanes" is available to flight crews and so must be classified Low. This can also occur if certain attributes are classified High, or if cover-stories are implemented. The Low-level view results in a subset of the High-level one. 


\subsection{ADDITIONS/DELETIONS}

Fortunately, for the database functions being considered (COUNT, MAX, MIN, SUM), there is a direct relationship between the value of the function and the size of the set operated on by that function. That is, $\operatorname{MAX}(\mathrm{A}) \leq$ MAX(B) if A $\leq B$, and similarly for COUNT and SUM (of positive values). For the functions MIN, and SUM (of negative values) there is an inverse relationship, so $f(A) \leq f(B)$ if $B \leq A$. Therefore, given $f_{1}$ and $\Theta$, we can determine whether $q_{3}\left(R_{L}\right)$ should be a subset or superset view. Indeed there are only four combinations, or cases, to consider.

Case 1. Assume:

(1) $f_{1}$ is COUNT, MAX or SUM (for positive values of $\mathrm{t}$ );

(2) $\Theta$ is $i, \leq$, =, or $\subseteq$.

(3) $q_{3}\left(R_{L}\right)$ is a subset view.

If adding a new tuple increases $f_{1}\left(q_{1}(R)\right)$, then $f_{1}\left(q_{3}\left(R_{L}\right)\right)$ will not increase by a greater amount, so $f_{1}\left(q_{3}\left(R_{L}\right)\right) \Omega f_{1}\left(q_{1}(R)\right)$ always holds.

For example: COUNT(SELECT(ID-Number FROM DB WHERE Plane_Type $=$ SR71 AND Mission_Classification $<$ Confidential)) $\leq$ COUNT(SELECT(Name FROM DB WHERE plane_type $=S R 71)$ ) will always hold, regardless of Mission_Classification.

Case 2. Assume:

(1) $f_{1}$ is MIN, or SUM (for negative values of $\mathrm{t}$ );

(2) $\Theta$ is $i, \geq,=$, ?.

(3) $q_{3}\left(R_{L}\right)$ is a subset view.

If adding a new tuple decreases $f_{1}\left(q_{1}(R)\right)$, then $f_{1}\left(q_{3}\left(R_{L}\right)\right)$ will not decrease by a greater amount, so $f_{1}\left(q_{3}\left(R_{L}\right)\right) \Omega f_{1}\left(q_{1}(R)\right)$ always holds.

For example: MIN(SELECT $\left(E m p \_P a y\right.$ FROM (DB WHERE Job = analyst)) $\geq \operatorname{MIN}\left(\operatorname{SELECT}\left(E m p \_\right.\right.$Pay FROM DB WHERE Job = analyst OR Job $=$ agent)) will always hold (assume "agent" records are classified higher than "analyst" records).

Case 3. Assume:

1) $f_{1}$ is COUNT, MAX or SUM (for positive values of $\mathrm{t}$ );

(2) $\Theta$ is $i, \geq,=$, ?.

(3) $q_{3}\left(R_{L}\right)$ is a superset view. 
Then an increase to $f_{1}\left(q_{3}\left(R_{L}\right)\right)$ will not always increase $f_{1}\left(q_{1}(R)\right)$, so $f_{1}\left(q_{3}\left(R_{L}\right)\right) \Omega f_{1}\left(q_{1}(R)\right)$ always holds.

For example: COUNT(SELECT(Name FROM DB )) $\geq$ COUNT(SELECT( Name FROM DB WHERE Real_Agency .eq. "CIA")) will always hold, regardless of the value of Real_Agency.

Case 4. Assume:

(1) $f_{1}$ is MIN, or SUM (for negative values of $\mathrm{t}$ );

(2) $\Theta$ is $i, \leq,=$, or $\subseteq$.

(3) $q_{3}\left(R_{L}\right)$ is a superset view.

Then if adding a new tuple decreases $f_{1}\left(q_{3}\left(R_{L}\right)\right)$ it will not always decrease $f_{1}\left(q_{1}(R)\right)$, so $f_{1}\left(q_{3}\left(R_{L}\right)\right) \Omega f_{1}\left(q_{1}(R)\right)$ always holds.

For example: MIN(SELECT(Mission_Cost FROM DB )) $\leq$ MIN(SELECT( Mission_Cost FROM DB WHERE Mission .eq. “spy”)) will always hold, regardless of the value of Mission.

For other combinations of $q_{3}, f_{1}$, and $\Theta$ (i.e. $f_{1}=$ COUNT, $\Theta \equiv \leq$, and $\left.q_{3}\left(R_{L}\right) \subseteq q_{1}(R)_{L}\right)$, there will exist a level-valid constraint which is sufficient to validate the multilevel constraint for tuple deletions. That is, for the complimentary conditions, tuples can be deleted (but not added) without checking the original multilevel constraint, although the multilevel constraint must still be checked if tuples are added to ensure that the new set is still small enough to satisfy the constraint. If $f=$ identity, $f\left(q_{1}(R)\right)=q_{1}(R)$ so $\left(q_{1}(R)\right)_{L}=q_{3}\left(R_{L}\right)$. In dealing with identity functions, we must establish both subset and superset conditions, so that $\left(q_{1}(R)\right) L=q_{3}\left(R_{L}\right)$.

\subsection{MODIFY OPERATIONS}

A "modify" operation cannot be handled as two separate operations but must be considered as an atomic transaction. The basic principles derived above must still apply, that is, the Low view will still be either a subset or a superset of the High view. However, it is no longer clear if the function $f_{1}$ is increasing or decreasing. In fact, this will normally depend upon the actual values for the attributes in the new and old copies of tuple t. For example, if $f_{1}=\mathrm{SUM}$, then $f_{1}$ is increasing when $\left|q_{3}(t)\right|>0$, and $f_{1}$ is decreasing when $\left|q_{3}(t)\right|<0$.

Table 16.1 presents these additional conditions which must be evaluated in order to determine the behavior of these functions. These conditions become simple tests for the validity of modifying tuples. In Table 16.1, $L=$ $f_{1}\left(q_{3}\left(R_{L}-t_{o} \bigcup t_{n}\right)\right) \Theta f_{2}\left(q_{2}(R)\right)_{L}$ is the constraint for modification of tuples $\left(t_{o} \rightarrow t_{n}\right)$ where the RHS of $f_{1}\left(q_{1}(R)\right) \Theta f_{2}\left(q_{2}(R)\right)$ is unaffected by tuple modification. 
Table 16.1 Level valid tuple modification constraints.

\begin{tabular}{|l|l|l|l|l|}
\hline \multicolumn{1}{|c|}{$\begin{array}{l}q_{3} \text { - Superset } \\
\left.\text { (i.e. } q_{1}(R) \subseteq q_{3}\left(R_{L}\right)\right)\end{array}$} & \multicolumn{3}{|c|}{$\begin{array}{l}q_{3} \text {-Subset } \\
\left.\text { (i.e. } q_{3}\left(R_{L}\right) \subseteq q_{1}(R)\right)\end{array}$} \\
\hline$f_{1} \mid \Theta\left(\begin{array}{l}\text { conditions on } \\
t_{o} \text { and } t_{n}\end{array}\right.$ & $f_{1}$ & $\Theta$ & $\begin{array}{l}\text { conditions on } \\
t_{o} \text { and } t_{n}\end{array}$ \\
\hline CNT $\mid$ all $\mid$ none & CNT $\mid$ all $\mid$ none \\
\hline MAX $|\geq,=|$ none & MAX $|\geq,=| q_{3}\left(t_{n}\right) \Theta q_{3}\left(t_{o}\right)$ \\
\hline MAX $|\leq,=| q_{3}\left(t_{n}\right) \Theta q_{3}\left(t_{o}\right)$ & MAX $|\leq,=|$ none \\
\hline SUM $|\leq,=| q_{3}\left(t_{o}\right)-q_{3}\left(t_{n}\right) \geq 0 \mid$ SUM $|\leq,=|$ none \\
\hline SUM $|\geq,=|$ none & SUM $|\geq,=| q_{3}\left(t_{o}\right)-q_{3}\left(t_{n}\right) \geq 0 \mid$ \\
\hline MIN $|\geq,=| q_{3}\left(t_{n}\right) \Theta q_{3}\left(t_{o}\right)$ & MIN $|\geq,=|$ none \\
\hline MAX $|\leq,=|$ none & MAX $|\leq,=| q_{3}\left(t_{n}\right) \Theta q_{3}\left(t_{o}\right)$ \\
\hline
\end{tabular}

\subsection{LIMITATIONS}

If any of the following are true we can make no statement concerning the existence of a level-valid constraint: (1) $q_{3}\left(R_{L}\right)$ is neither a subset nor a superset of $q_{1}(R)$; (2) $f_{1}$ is not monotonic with respect to set sizes; or (3) $\Theta$ does not specify a partial order relation. This does not mean that such level-valid constraints do not exist, merely that their existence cannot be proven by this mechanism. Usually heuristic analysis is required.

Fortunately, the situation, $f_{1}\left(q_{3}\left(R_{L}\right)\right)=f_{1}\left(q_{1}(R)\right)_{L}$, is quite common and satisfies both Superset Class and Subset Class requirements. In such a case, level-valid constraints will exist for both addition and deletion of tuples.

\subsection{CONSTRAINT VIEW DERIVATION}

We have two choices for views (subset or superset), two choices for functions (increasing or decreasing), and only two real choices for comparators $(<$, $>$ ). In practice, only the view in the level-valid constraint will differ from the one in the multilevel valid constraint. We will examine each choice in more detail and summarize the results as rules that may be applied to the multilevel view to derive the level-valid view. In the following discussion, we consider each view $q(R)$ as being decomposed into the unary and binary func- 
tions available in a relational database. In this approach, the "q" consists only of "select/project" operations, while the " $R$ " may represent a base relation or one formed by a Cartesian product, complement, or union.

3.6.1 The Superset View: $q_{1}(R) \subseteq q_{3}\left(R_{L}\right)$. To derive a level-valid superset, $q_{3}\left(R_{L}\right)$, of a multilevel view, $q_{1}(R)$, we must define a new level-valid view, operating only on data at level $L$ or below, that still contains all the tuples originally in $q_{1}(R)$. The first thing to note is that, while $q_{1}(R)$ references High classified information, a level-valid view cannot actually return High classified information. Since we must keep all tuples that satisfy $q_{1}(R)$ and these must all be classified $L$ or below, $q_{1}$ cannot contain "project" clauses referring to higher classified attributes. This leads to the first rule:

Superset Rule 1: For Superset Class situations, a level-valid constraint is not derivable if $q_{1}$ contains a project clause returning High classified attributes.

Generally, if $q_{1}$ does not contain High project operations, we can set $q_{3}=q_{1}$, but eliminate select clauses referring to High attributes. Eliminating select clauses does not eliminate any tuples, the new query will simply have fewer restrictions and additional tuples will satisfy it. This is why $q_{3}$ is regarded as specifying a superset of the original query, $q_{1}$.

Superset Rule 2: Eliminate select clauses relating to High attributes.

We now have two rules explaining how $q_{3}$ differs from $q_{1}$. We must also know if the relation, $R$, needs to be changed. The project and select operations apply to a database relation. There are three additional operations that may be used to define that relation: Cartesian product; union; and complementation.

(a) Cartesian Product: Consider $q_{1}(R)$ where $R=R_{L} \times R_{H}$ with $R_{H}$ being classified High. Tuples formed via Cartesian products will contain some Low classified attributes and some High classified attributes. However, Since $q_{3}$ contains no clauses, either project or select, referring to High classified attributes, the view $q_{3}\left(R_{L} \times R_{H}\right)$ cannot contain any tuples with High classified attributes. The tuples satisfying the query therefore cannot contain more information than those tuples satisfying $q_{3}\left(R_{L}\right)$, and hence could be formed directly from $R_{L}$. It is possible that the Cartesian product could create duplicate tuples, but these would be eliminated in the view, so $q_{3}\left(R_{L} \times R_{H}\right) \subseteq q_{3}\left(R_{L}\right)$. 
(b) Union: Consider $q_{1}(R)$ where $R=R_{L} \bigcup R_{H}$. Suppose that $q_{1}\left(R_{L}-\right.$ $\left.R_{H}\right)=q_{1}\left(R_{L}\right) \bigcup q_{1}\left(R_{H}\right)$, and $q_{1}\left(R_{H}\right) \neq \emptyset$. Then $q_{1}$ contains project clauses relating to High classified attributes, and we cannot generate a superset case.

(c) Complementation: Since $R_{L}$ and $R_{H}$ are disjoint, they have no tuples in common. Consider first $q_{1}\left(R_{L}-R_{H}\right)$. But $q_{1}\left(R_{L}-R_{H}\right)=q_{1}\left(R_{L}\right)-q_{1}\left(R_{H}\right)$, which we replace with: $q_{3}\left(R_{L}\right)-q_{3}\left(R_{H}\right)$. So $q_{3}\left(R_{L}\right)$ is larger than $q_{1}\left(R_{L}-R_{H}\right)$, and we have a superset case. Therefore, $R_{L}-R_{H}$ may be replaced with $R_{L}$ to form a superset case. Consider now $q_{1}\left(R_{H}-R_{L}\right)$. This reduces to: $q_{3}\left(R_{H}\right)-q_{3}\left(R_{L}\right)$, but $q_{3}\left(R_{H}\right)=\emptyset$ (the null set), so "removing tuples" is not defined, and we must set $q_{3}\left(R_{H}-R_{L}\right)=\emptyset$. So, in cases other than High complementation, the High relation may simply be removed from consideration.

\section{Superset Rule 3:}

$$
\begin{aligned}
& \text { If } R=R_{L} \times R_{H}, \text { or } R=R_{L}-R_{H}, \text { set } R=R_{L} . \\
& \text { If } R=R_{H}-R_{L}, \text { set } q_{3}\left(R_{L}\right)=\emptyset .
\end{aligned}
$$

Similar rules may be derived for subset views; $q_{3}\left(R_{L}\right) \subseteq q_{1}(R)$.

3.6.2 Example 3:. Assume a common database consisting of two tables: EMP (employee, classified $L$ ) MGR (managers, classified $H$ ). The constraint "if an employee's manager makes less than \$ 50k then the employee must make less than $\$ 25 \mathrm{k}$ " (i.e. higher paid managers are allowed to hire higher paid subordinates). Assume that the employee record defines the salary, Salary, and the employee's manager, Mname. Denote as $T$ the "table" corresponding to the single employee tuple, $t$. The multilevel constraint for addition of an employee tuple then is:

$M=$ (Select $t$. Salary from $T, M G R$ where $T . M n a m e=M G R . M n a m e$ and $(M G R$.Salary $\leq \$ 50 K))<\$ 25 k$

Or, by separating out the natural join, this is equivalent to: $M=($ Select $t$. Salary from $(T, M G R$ where $T . M n a m e=M G R . M n a m e)$ where $(M G R$. Salary $<\$ 50 k)<\$ 25 k$

Since we are dealing with an identity function, it may be considered either a subset or a superset case. We may eliminate the natural join and the High classified select clause, giving the level-valid constraint for an employee tuple as: 
$L=($ Select $t$. Salary from $T) ; \$ 25 \mathrm{k}$

Note that this constraint also satisfies the tuple deletion conditions, so the database does not have to be checked for consistency if employees making less than $\$ 25 \mathrm{k}$ are either hired or fired. Although the set of rules appears like a complicated and disconnected list of special cases and disconnected rules, in reality it is no more complicated than any other algebraic system. The process of generating a level-valid constraint is similar to other algebraic reduction procedures. Simply apply the right rule at the right time.

\section{CONCLUSIONS}

We have shown that many multilevel integrity constraints can be transformed into multiple level-valid constraints whose satisfaction is sufficient to ensure that the original multilevel constraint is also satisfied. The level-valid constraints are free from signaling channels.

This transformation is facilitated by devising a simple, powerful, yet intuitive method of expressing constraints as the relationship between the values of aggregate functions applied to views. In many cases it is possible to transform the original multilevel constraint into multiple level-valid constraints whose satisfaction is sufficient to ensure that the original is also satisfied. In transforming the multilevel constraint we have two possibilities: (1) the aggregate function remains valid if applied against a subset of the original tuples (i.e. the maximum value attained in a set will be at least as great as the maximum value attained in any subset) or (2) the aggregate function remains valid if applied against a superset of the original tuples (i.e. the maximum value attained in a set will be no greater than the maximum value attained in any superset).

Within this general solution, we can actually derive level-valid constraints for the common database operations and functions. We describe the procedures for generating the needed set of tuples as a view at level $L$ or below, having the desired relationship (that is, being either a subset or a superset) to the view specified in the constraint. The generation of this new view is straightforward, but the techniques vary depending upon the relational algebra operations being used.

The techniques may be generalized for use with transactions, or constraints expressed as triggers, not simply the tuple additions/deletions considered already. These new level-valid constraints do not solve the entire problem, and occasionally tuple modifications will have to rely upon trusted methods as are commonly used in current implementations. However, the situation is never more complicated, and level-valid constraints are usually simpler and more straightforward than the trusted process technique. 


\section{References}

[1] Akl, S.G. and Denning, D.E. (1987). Checking classification constraints for consistency and completeness. Proceedings of the IEEE Symposium on Security and Privacy, pp. 196-201.

[2] Burns, R.K. (1990). Referential secrecy. Proceedings of the 1990 IEEE Symposium on Security and Privacy, pp. 133-142.

[3] Denning, D.E., Lunt, T., Neumann, P. and Schell, R. (1986). Secure Distributed Data Views - Security Policy and Interpretation for a Class A1 Multilevel Secure Relational Database System, SRI International.

[4] Gupta, A., and Widom, J. (1993). Local verification of global integrity constraints in distributed databases. Proceedings of the 1993 ACM SIGMOD International Conference on Management of Data, pp. 49-58.

[5] Jajodia, S., Marks, D. and Bertino, E. (1995). Maintaining secrecy and integrity in multilevel databases: A practical approach. Proceedings of the Eighteenth National Information Systems Security Conference, pp. 37-49.

[6] Maimone, W.R., and Allen, R. (1991). Methods for resolving the security vs. integrity conflict. Proceedings of the Fourth RADC Multilevel Database Security Workshop, pp. 55-59.

[7] Meadows, C., and Jajodia, S. (1998). Integrity versus security in multilevel secure databases. Database Security: Status and Prospects (ed. Carl E. Landwehr), North-Holland, pp. 89-101.

[8] Qian, X. (1994). Inference channel-free integrity constraints in multilevel relational databases. Proceedings if the 1994 IEEE Symposium on Security and Privacy, pp. 158-167.

[9] Sandhu, R.S., and Jajodia, S. (1993). Referential integrity in multilevel secure databases. Proceedings of the Sixteenth National Computer Security Conference, pp. 39-52. 